\title{
Specimen gripping effects in composites fatigue testing - Concerns from initial investigation
}

\author{
P. B. S. Bailey ${ }^{1 *}$, A. D. Lafferty ${ }^{2}$ \\ ${ }^{1}$ Instron Materials Testing, Coronation Road, HP12 3SY High Wycombe, United Kingdom \\ ${ }^{2}$ Department of Materials Science and Engineering, University of Sheffield, Broad Lane, Sheffield, United Kingdom
}

Received 14 August 2014; accepted in revised form 10 December 2014

\begin{abstract}
This paper presents the findings of an initial investigation into effects of end tabs and gripping force on specimens for fatigue testing of structural composites. Live measurement of specimen compliance comparing extensometer measurements with grip displacement showed the impact of gripping on the dynamic behavior of the specimen. Use of thermal imaging enabled assessment of how load is introduced and carried along the specimen.

The effect of grip pressure and comparative merits of popular solutions are examined in the context of fatigue testing of composites. Although for static tests these potential issues have been investigated extensively throughout the history of composites research, interest has only recently fallen upon their effects on dynamic loading. Data collected show that the gripping pressure at the ends of the specimen can affect results more significantly than may be anticipated. These early results suggest that solutions which are well established for quasi-static testing may cause problems in cyclic tests.
\end{abstract}

Keywords: polymer composites, fracture and fatigue, material testing, thermography, stress concentration

\section{Introduction}

\subsection{Background}

Having regularly received enquiries regarding appropriate gripping forces for composite test coupons, and regarding specimen failures not only at the jaw face, but within the tabbed and gripped section, the authors sought to clarify the origins of these problems. The results of this initial investigation were considered of significant concern to the composites testing community, so are published within this paper while a wider investigation is ongoing.

Instron (Dynamic Systems) noticed that a rise in commercial demand for composites fatigue data started in the mid 2000's and this has continued to grow unabated since then. This was originally driven by the wind turbine industry, where structures experience significant cyclic strain over a long life cycle. By comparison, academic research interest in com- posites fatigue was particularly strong during the 1980 s through into the mid-90s, along with all aspects of mechanical characterization of composites. Although numbers of publications remained consistent ever since, the topic seemed to become a less popular research and conference topic until around 2010, since when there has been a rapid increase in activity, primarily driven by the aerospace and automotive industries.

It has long been known and demonstrated that gripping methods can significantly affect both the value and the consistency of results in mechanical testing of composites [1,2] and certain test practices have become commonly accepted for quasi-static tests. Nonetheless, it is interesting to note that ASTM D3039 for tensile testing of composites remarks that 'Design of mechanical test coupons, especially those using end tabs, remains to a large extent an art rather

\footnotetext{
${ }^{*}$ Corresponding author, e-mail: peter bailey@instron.com (C) BME-PT
} 
than a science, with no industry consensus on how to approach the engineering of the gripping interface' [3]. With growing drive to understand the dynamic behavior of composites $[4,5]$, an increasing number of experimentalists are finding it necessary to revisit their previous methodologies for specimen preparation.

\subsection{Specimen and gripping designs}

Straight sided tensile specimens with composite end tabs are now the accepted basic standard for tensile testing of composites [3, 4]. Unfortunately, in a majority of scenarios, where specimens comprise a large amount of on-axis reinforcement, these typically fail at, or only just beyond, the end of the tab $[1,4,7]$. Qualitatively, the analyst knows that this will be the region of highest constraint, and therefore the highest stress concentration, therefore physics dictates that the specimen must fail in that region, unless there is some other, more significant stress raiser or defect. This has been demonstrated quite elegantly, for a variety of configurations, using finite element analysis [8] with some straightforward mechanical tests provided to confirm the predictions. A number of workers examined tapered end-tabs $[1,9,10]$ but it appears that results varied from no noticeable difference in failure, through to complete success in establishing failure away from the grips. Whatsoever the efficacy of this technique, tapered tabs are now suggested in international standards $[3,6]$, but anecdotally, many laboratory operators look on them unfavourably due to the even more laborious preparation required. Early recommendations $[1,11]$ proposed a variety of tabs including different configurations of fibre reinforced tab material, but also compliant abrasive pads, and aluminium sheet. Only glass fibre reinforced polymer matrix tabs have been carried forward to current international standards, although some researchers still use aluminium tabs and consider them easier and more reliable to prepare.

Several researchers experimented with a gently radiused gauge section, with the goal of forcing failure into a region of known and consistent stress state (away from the grips) as is well established for metals and plastics testing. Some work is published in open literature [12-14], but the authors have also been privileged to be shown similar results by industrial partners who have undertaken similar which remains unpublished. In certain respects, the pub- lished work shows a basic level of success, in terms of generating failure within the designated gauge section. However, this does not appear to significantly improve repeatability; it is unclear whether the dominant factor in variability is therefore related to the material or to the specimen preparation. More importantly, a non-critical failure is observed in many tests presented; prior to complete tensile rupture, the specimens develop a shear failure, parallel to the tensile axis, aligned with the edges of the narrowest point of the gauge section. This is particularly concerning for fatigue analyses, where considerable insight may be obtained by calculating the dynamic behavior of the specimen (for example stiffness, modulus, loss tangent, cyclic work done); clearly if the geometry of the load carrying section of the specimen changes, then any such calculations will show a change in output, but will no longer give a comparable measure of the material performance. This might be circumvented by use of strain gauges bonded directly to the specimen, but this raises the risk of not measuring a sufficiently large surface area to be representative, especially with commercial demand for use of large fibre tows and woven reinforcements.

By comparison, the use of open-hole specimens for fatigue does appear to reliably force failure into a defined location, and although this was demonstrated some time ago $[15,16]$, it was not developed into an international standard until 2011 [17] and is as yet only used by a small number of workers. Early work on fatigue damage in composites also examined the possibility of using on-axis uni-directionally reinforced material with an elongate, transverse, open hole. Growth of the longitudinal fractures propagating from the edges of the hole (as seen from the edges of the radii on dumbbell specimens discussed above) was evaluated, with some success, but the concept does not appear to have been advanced beyond the original work by Spearing and Beaumont [18].

\subsection{Clamping pressure}

Historically, mechanical testing practitioners have often used a 'rule of thumb' that the gripping force on the ends of a typical specimen should be approximately 10 to $15 \%$ larger than the maximum axial test load to be applied. Although at present the authors cannot identify the origins of this guideline, it seems highly probable that it originates in testing of metals coupons, where specimens have more compliant 
surfaces making them easier to indent and grip. Furthermore a majority of tensile testing on metals and polymers utilizes wasted specimens where the gripped section is wider than the gauge length. It is the authors' opinion that this level of clamping pressure is usually inadequate for typical, parallel sided specimens, of structural composites.

\section{Materials and experimental pocedure 2.1. Specimen preparation}

Tests were conducted on E-glass fibre reinforced epoxy resin sheet, prepared by wet lay-up and vacuum consolidation.

Industrial grade, low temperature curing epoxy resin was used, with fibre in the form of E-glass $280 \mathrm{gsm}$ non-crimp fabric. All specimens were produced in one batch, using the same batches of raw materials. The raw materials used in this initial work were generic industrial products, as supplied by East Coast Fibre Supplies, who do not offer further certification of composition or mechanical performance.

Material was produced by wet laying 6 plies of noncrimp fabric between polyamide peel-ply release film, on a polished steel caul plate, then vacuum consolidating at better than 0.95 bar until gelled. The consolidated material was moved to an oven (still on caul plate within bag and under vacuum) for final cure at $60^{\circ} \mathrm{C}$ for 4 hours.

Specimens were prepared in accordance with ISO 257 [6], cut by diamond wheel, with parallel gauge section of 25 by $150 \mathrm{~mm}$. The $50 \mathrm{~mm}$ long gripped section was adjusted for comparison between: tabs of cross-plied, glass fibre reinforced epoxy, $2 \mathrm{~mm}$ thick $[6,11]$; tabs of aluminium sheet, $2 \mathrm{~mm}$ thick [11]; no bonded tab [1].

Where used, the adhesive was the same composition and subject to the same cure as the matrix material. Bonded surfaces were thoroughly solvent washed with acetone, then abraded, then re-washed and allowed to dry, prior to bonding. Composite surfaces were abraded using bonded abrasive paper; aluminium tabs were grit blasted with clean silica sand.

\subsection{Mechanical testing}

Specimens were tested using an Instron 8801MT servohydraulic materials testing system, rated to $100 \mathrm{kN}$ peak dynamic load, with a control system capable of $10 \mathrm{kHz}$ data acquisition and loop closure rate. Direct strain measurements were taken using an Instron dynamic extensometer with a $25 \mathrm{~mm}$ gauge length (a strain gauged, clip-on extensometer, calibrated at $2 \%$ of a $12.5 \mathrm{~mm}$ gauge length, manufacturer's part No. 2620-602), at the mid-point of the free length of each specimen. Specimens were clamped using hydraulically tensioned, wedge acting grips (rated for up to $100 \mathrm{kN}$ axial load), with a regulated acting hydraulic pressure.

For the purposes of this study, tests were carried out in tension-tension mode, at a single loading level. After tuning the control system, each specimen was subjected to a cyclic load at $5 \mathrm{~Hz}$, at a loading ratio of 0.1 , to a peak stress equating to $50 \%$ failure strength. After an initial bedding-in period of 1000 cycles, to overcome the initial phase of rapid damage accumulation, the hydraulic pressure was varied up to 200 bar (supply pressure is nominally 207 bar), in increments of $20 \mathrm{bar}$, to apply a range of clamping stresses on the gripped section of the specimen.

For reference, the peak tensile load in these tests was $15 \mathrm{kN}$, which according to the guidance for gripping of metals (discussed earlier) would correspond to a gripping stress of just $14.3 \mathrm{MPa}$; no data point was taken for this value since the required hydraulic pressure is so low that it is not always sufficient to overcome mass and static friction on opening or closing the grips.

Key parameters of the dynamic behavior of the specimen (including phase angle, stiffness and modulus, for in-phase, out-of-phase, and complex behavior) were calculated live, for each cycle, by the test control software, using simulated sine wave correlation algorithms.

\subsection{Infrared thermography}

It is fairly well known that composites under cyclic loading are subject to significant self-heating effects $[14,19-21]$, through a combination of viscous heating and energy release through micro-fractures. Obviously this presents other experimental concerns which have been discussed elsewhere, but it facilitates identification of where strain and damage are being introduced [22].

An Optris PI450 bolometer thermal imaging camera was used to examine the edge of the specimen as it passed into the gripped section, and at the end of each test an image was captured and line scan data extracted along the centerline. Data are presented as a temperature increase profile; after subtraction of a baseline profile of the gripped specimen, prior to any loading. 
For the purposes of this initial investigation, it was not deemed necessary to apply a constant emissivity coating, since all surfaces of interest were of the same material, meaning that the absolute surface temperatures calculated are of uncertain accuracy, but over the small temperature range present in these tests, the relative changes of interest are highly reliable. Surface temperature calculations were based on an emissivity of 0.96 , typical for polymer matrix composites.

Specimen heating effects are directly linked to applied strain amplitude and to progressive damage accumulation, so the locations of more severely loaded regions of the specimen may be inferred from the temperature distribution. This approach is distinct from thermo-elastic stress analysis [23, 24]
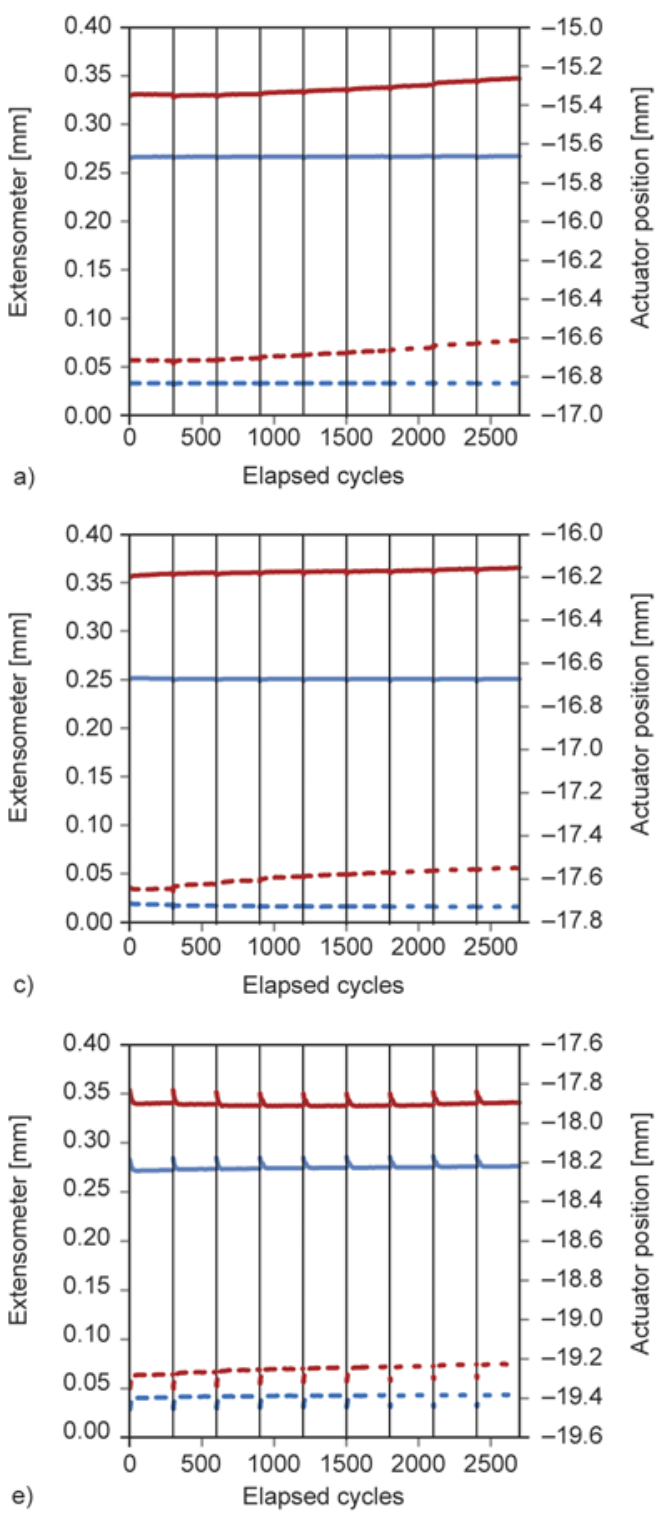

and the two must not be confused. TSA is a differential technique for evaluating the relative magnitude of the change in stress distribution between two loading states of a test piece subject to purely elastic load; in cyclic tests (on composites) it can only reliably be applied between two loading points within a single cycle over a relatively small stress range. The approach used for this paper is effectively measuring the cumulative energy dissipation in the material over a large number of cycles, from which a semiquantitative measure of strain amplitude is inferred.

\section{Results and discussion}

\subsection{Raw data}

Raw data for strain measurements for each specimen is presented in Figure 1. Since this is a fatigue
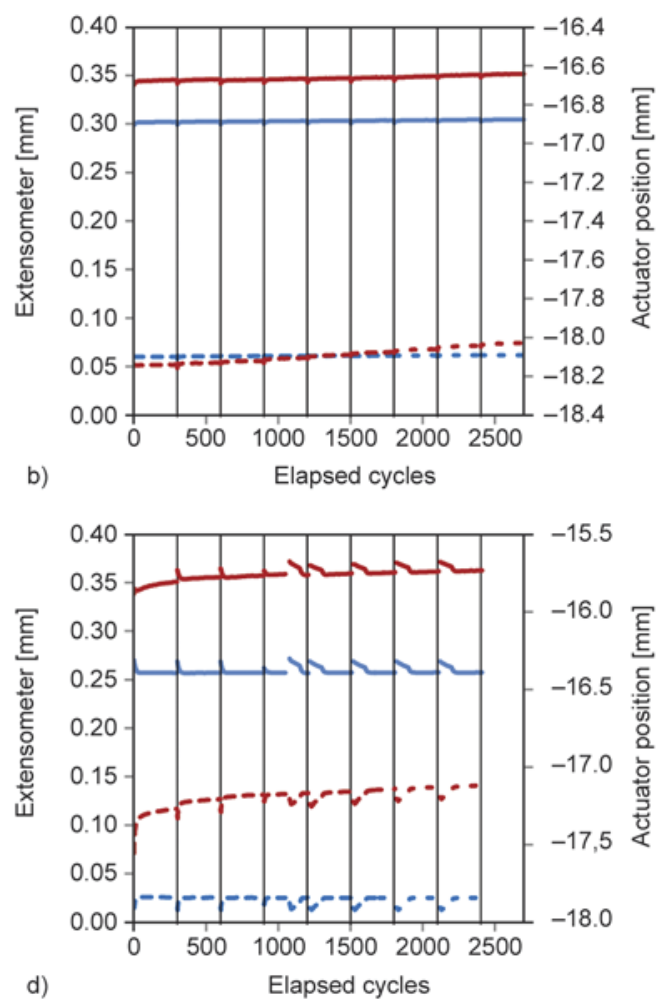

Figure 1. Peak strain from actuator displacement or extensometer reading vs elapsed cycles; vertical lines denote the timing of increments in gripping stress, a) GFRE tab, b) aluminium tab, c) no tab, d) abrasive cloth, e) SiC coated jaws 

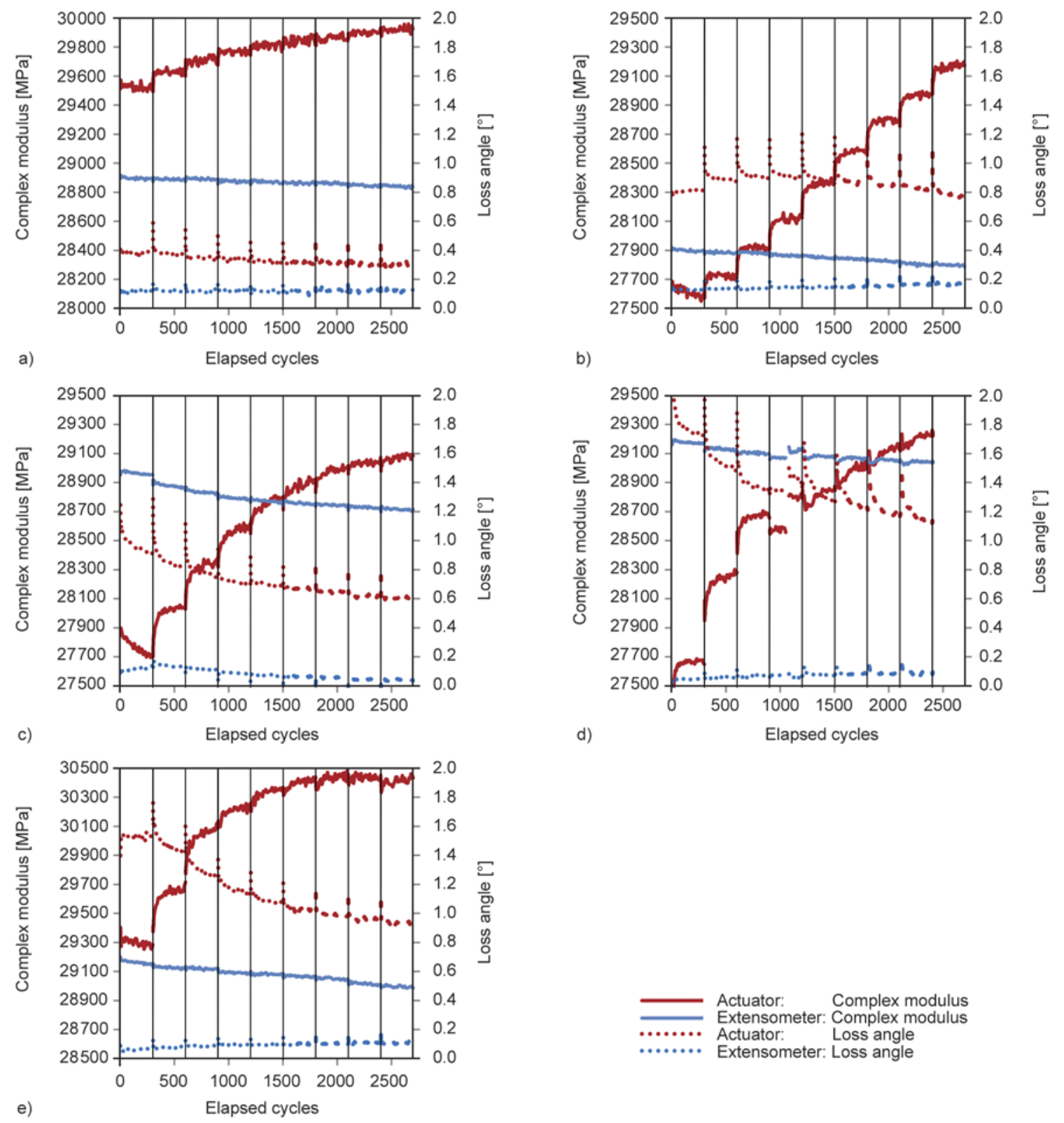

Figure 2. Calculated complex modulus from actuator displacement or extensometer reading vs elapsed cycles; vertical lines denote the timing of increments in gripping stress, a) GFRE tab, b) aluminium tab, c) no tab, d) abrasive cloth, e) $\mathrm{SiC}$ coated jaws

test, the specimen behavior does change with elapsed cycles, hence these charts are presented with cycles on the $x$-axis; vertical lines are not gridlines, but instead denote each point at which the hydraulic pressure to the grips was increased, by a step or 20 bar. The values of dynamic modulus and phase angle calculated live with each cycle are plotted in a similar manner in Figure 2.

\subsection{Complex dynamic modulus}

Under ideal conditions it is assumed that the specimen would be gripped such that there is no slipping of the specimen within the jaws, that load is intro- duced evenly without stress concentrations, and that the stiffness of the grip body and actuator are greatly above that of the specimen under test. Since our current standards for composite test coupons are straight and parallel sided, the result of this would be that any geometry-independent measurement of stiffness would ideally be identical when calculated from grip separation or extensometer respectively. Practically, this is clearly unrealistic, but many workers utilize grip separation to measure strain, for a variety of reasons. For this reason, the authors and many other sources frequently emphasize the importance of strain measurement on an appropriate gauge length. 


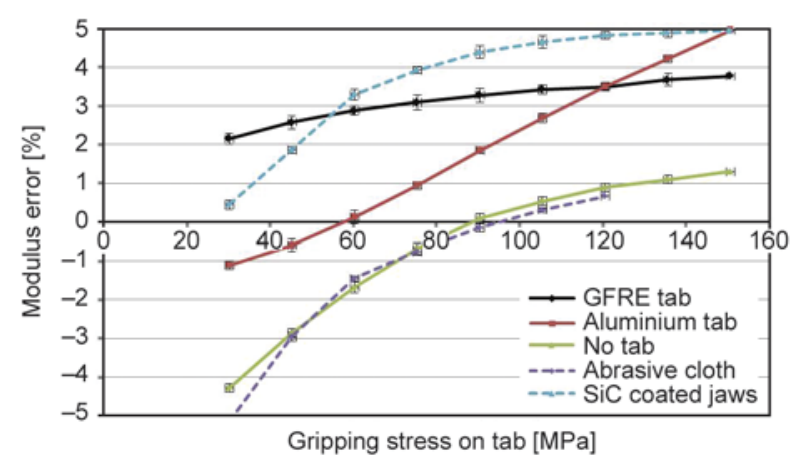

Figure 3. Error in measured complex modulus at grip vs gripping stress on tab (based on actuator displacement compared with extensometer measurement)

Figure 3 shows the error in complex modulus generated by imperfect gripping, and how it varies with the combination of clamping force and tab material. Each data point is the average of the percentage difference between 20 pairs of data points; 'error bars' on each data point indicate the maximum variation from mean.

The implication of a negative error is that there is significant shear or slippage within the gripped section; the effect of this is equivalent to a specimen with lower modulus or a larger gauge length. By contrast, positive errors suggest a degree of overconstraint on the specimen, causing the ends of the gauge length to exhibit greater on-axis stiffness. Logically it is also possible for a specimen to suffer from both effects at the same time; experiencing some slippage on the loading axis, while the ends of the gauge length are still subjected to a degree of over-constraint. For this reason, it is difficult to infer specific causality solely from the difference in extension or derived calculations, but it is the first step in identifying a problem.

On this basis it is postulated that the 'traditional' method of using cross-ply composite tabs results in noticeable over-constraint; potentially this is caused simply by the fact that the bonded tabs themselves apply considerable transverse constraint. Furthermore that this effect dominates compared with the small degree of slipping, which is known to happen at very low grip pressures through the evidence of abraded material which is found stuck in the serrations or the jaw face.

Conversely, the use of tab-less specimens (whether directly clamped in serrated, hardened steel jaws, or using the recommended layer of abrasive cloth) must give a significant degree of slipping or not clamp the full grip length. Using the same type of specimen with a silicon carbide bonded into the metal jaw surface still showed high sensitivity to clamping force, but surprisingly shifted the errors to a positive domain.

Finally, the use of aluminium tab material seems to be quite effective in achieving only a small error, without the need for high clamping forces which increase the risk of stress concentrations at the end of the gripped section.

\subsection{Loss angle}

Figure 4 shows the additional component of loss angle introduced at the grip, as compared with that at the extensometer measurement. Each data point is the average of the difference between 20 pairs of data points; 'error bars' on each data point indicate the maximum variation from mean. Loss angle (usually termed $\delta$, or expressed as $\tan \delta$ ) can be used as a measure of the damping in a system. Since it is known that the specimen material is moderately consistent for its whole length, it must be assumed that a phase difference between the two displacement measurements is introduced by slipping or shear within the gripping assembly.

This appears to confirm the earlier suspicion that the composite tabs are held quite effectively, while the un-tabbed specimens experience more significant slippage.

It is interesting to note that the specimen with aluminium tabs introduced a similar degree of lag to the untabbed specimen, but probably for different reasons. With the untabbed specimen this seems very likely to be related to slippage, but the aluminium tabs appeared securely gripped throughout, so their contribution may come from shear within the metal. It was not possible to use digital image correlation during this study to determine the actual displacement

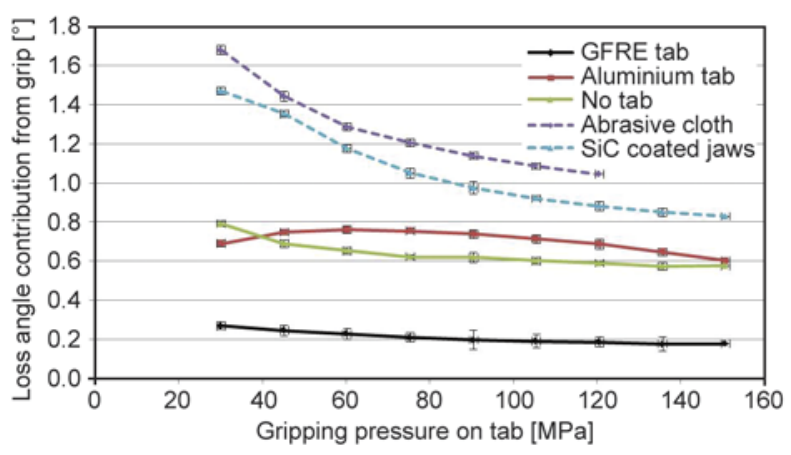

Figure 4. Additional phase lag introduced in grip vs gripping pressure (phase angle at extensometer for all tests was within $0.10 \pm 0.05^{\circ}$ ) 
fields, but the authors intend to pursue this in further work to resolve these questions.

\subsection{Temperature distribution}

Figure 5 shows thermal images of the 5 different tab/ grip configurations. A localized heating effect near the end of the grip is particularly obvious on the specimen with conventional composite tabs.

It is very clear that in the standard tabbed specimens, there is a very high degree of heating just within the end of the gripped section. The specimen gripped with un-bonded abrasive cloth behaved similarly. Using integral abrasive surfaces on the jaws, without tabs, seemed to reduce the sharp peak in heating, but caused more heat introduction further back into the grip, possibly due to sliding and abrasion. By contrast, specimens without tabs, or with aluminium tabs, show very gentle transitions from gripped to free length.

The specimens without any tabs and with aluminium tabs have remained much cooler that the others, probably due to much better conduction extracting more rapidly the heat which is generated. Since fatigue performance of composites is necessarily affected by temperature, due to the nature of the matrix materials, this would suggests that fatigue test specimens are doubly likely to fail at the grip, since the material near the end of the tab is both more highly stressed and at higher temperature.

Figure 6 plots the increase in surface temperature along the center-line of these specimens after cyclic loading

It is very clear that in the standard, composite-tabbed specimen, there is a very high degree of heating just within the end of the gripped section. Despite earlier

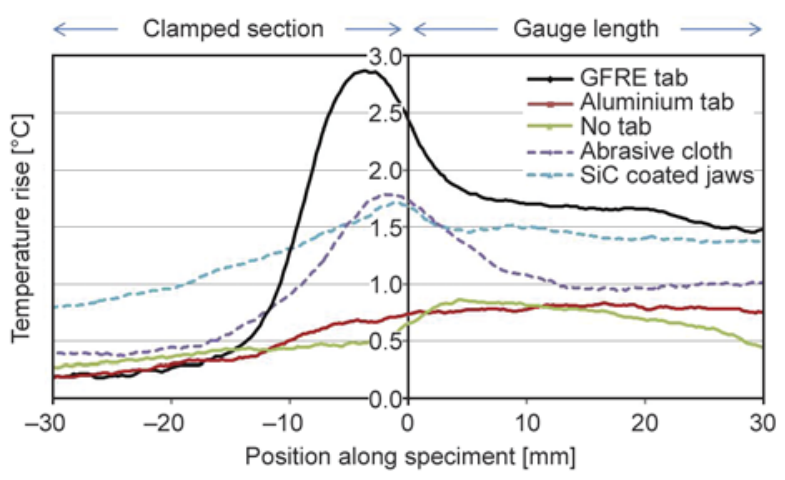

Figure 6. Temperature profiles along specimen edge as it enters the grip

indications (see section 3.4) that the tab-less specimen gripped with un-bonded abrasive cloth suffers significant slippage, the thermal activity in this configuration is very similar to the standard tabbed specimen. The implication of this is that the load introduction and damage localization is very similar between these two configurations. It may be argued that this is not an unexpected outcome, since these methods were established as moderately reliable and comparable early on in quasi-static test development for composites [1]; this would imply that the induced mechanical behavior of the specimen must be congruent in order to achieve similar results.

By contrast, specimens without tabs, or with aluminium tabs, show very gentle transitions from gripped to free length. It must be recognized that these specimens have a more direct connection to the metal jaws, through which heat might be conducted away into the thermal mass of the grips. Although this would surely suppress the magnitude of temperature rise, any significant local heating effects should still be apparent. It would appear that the severity of local

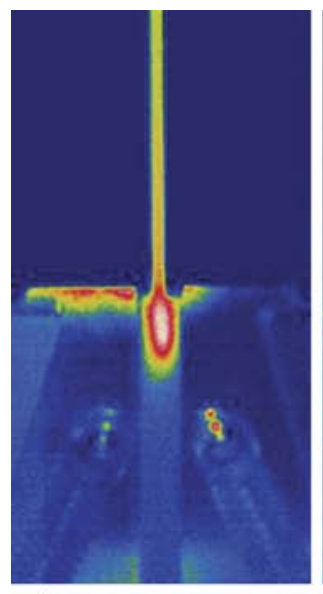

a)

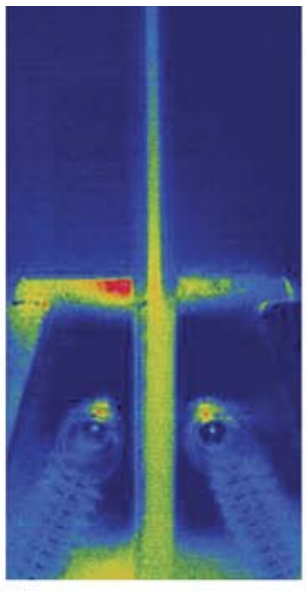

b)

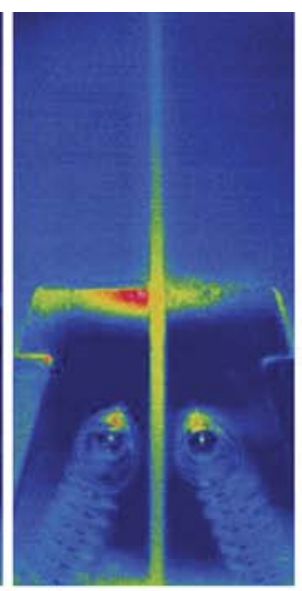

c)

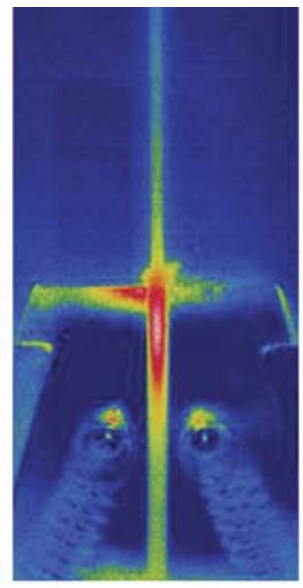

d)

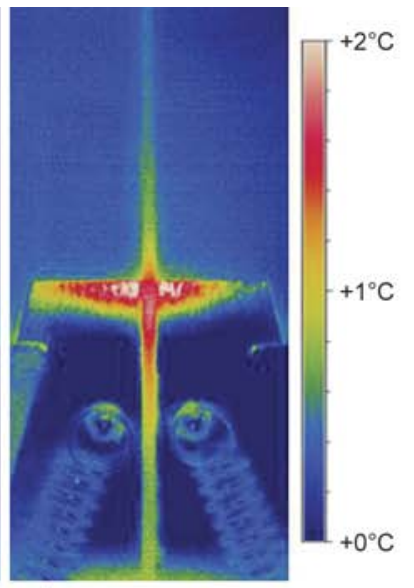

e)

Figure 5. Thermal images of the specimen edge after cyclic loading. (a) GFRE tab; (b) Aluminium tab; (c) Tab-less; (d) Tab-less with abrasive cloth; (e) Tab-less in SiC fused jaws. 
heating and grip-induced damage is reduced in these solutions, which do not conform to current international standards.

Using jaw faces with a rough silicon carbide surface, fused directly to the steel, with a tab-less specimen, seemed to reduce the sharp peak in heating, but caused more heat introduction further back within the grip. This may be due to sliding and abrasion of the surface, as previously discussed, which might reasonably overwhelm the better conductive path to extract heat into the jaws.

The authors have been unable to identify any other experimental analysis of stress or strain concentrations in the gripping region of composite test coupons for in-plane tensile or compressive property measurement. However, a survey of stress concentrations within the gripped section was conducted by De Baere et al. [8], using finite element modelling, for a fairly extensive range of tab and grip permutations. For GRFE tabbed specimens, the temperature rise illustrated by the line scans presented in Figure 6 seem to correspond well with the calculated stress concentrations along the specimen presented in that study. For aluminium tabbed specimens the computational method anticipated a small peak in stress concentration near the end of the tabs, which is not observed in the experimental data here, although this effect may be masked by the much better heat conduction along and out of the specimen, as already mentioned. De Baere et al. [8] did not examine untabbed specimens.

During the tests, it was also noted that the temperature distribution appears to change with the applied
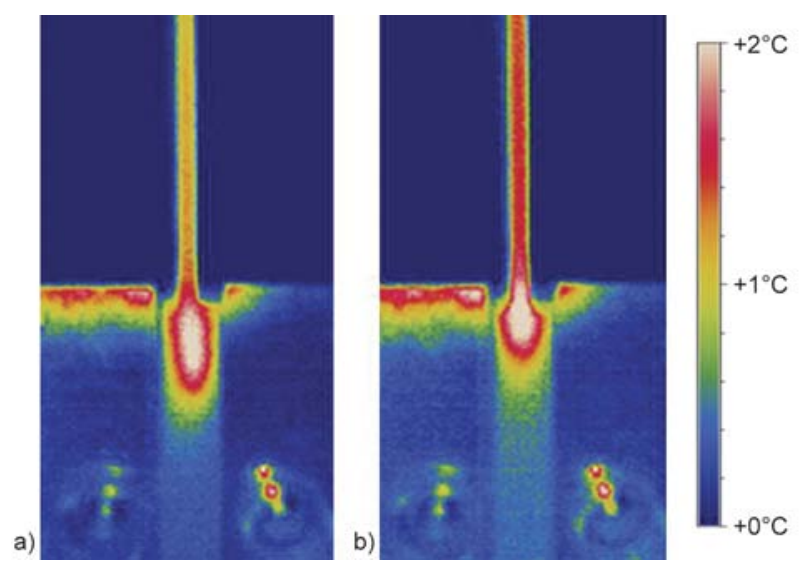

Figure 7. Temperature distribution on GFRE tab specimen (a) after 1600 cycles with $30 \mathrm{MPa}$ clamping stress and (b) after a further 2100 cycles at $120 \mathrm{MPa}$ clamping stress clamping stress. As shown in Figure 7, qualitatively speaking, higher clamping stress seems to push the localized heating into a tighter region, closer to the end of the gripped section, or just beyond it on some tab-less specimens. At the time of writing, insufficient data was available to draw any reliable conclusions without further testing.

\section{Conclusions}

The implication of these data is that, at least in cyclic testing of composites, the standard method of gripping composite coupons can easily cause damage and failure outside the gauge length, under un-representative loading conditions. This initial study gives some clues for how to locate and reduce the problem, and the findings are in agreement with computational models of stress concentration, as well as anecdotal evidence from a significant number of workers who regularly observe failure within the gripped section of composite fatigue test coupons.

The short case study presented here is based on a small sample size, but the indications are that there is a need to reconsider the best methods for gripping composite specimens for fatigue loading. The authors intend to verify this on a larger sample, and using several other representative materials.

\section{References}

[1] Hart-Smith L. J.: Generation of higher composite material allowables using improved test coupons. in 'Proceedings of the $36^{\text {th }}$ International SAMPE Symposium, San Diego, USA' 1029-1045 (1991).

[2] Hojo M., Sawada Y., Miyairi H.: Influence of clamping method on tensile properties of unidirectional CFRP in $0^{\circ}$ and $90^{\circ}$ directions - Round Robin activity for international standardization in Japan. Composites, 25, 786-796 (1994).

DOI: 10.1016/0010-4361(94)90139-2

[3] ASTM D3039/D3039M-14: Standard test method for tensile properties of polymer matrix composite materials (2014).

[4] Becerra N.: Flight mission. Testing, testing. Materials World, 22, 26-27 (2014).

[5] Bailey P. B. S.: Growth in dynamic testing market for composites. Advanced Materials and Processes, 172, 17-21 (2014).

[6] BS EN ISO 527-5: Plastics. Determination of tensile properties. Test conditions for unidirectional fibre-reinforced plastic composites (2009).

[7] Morrell, R., McCartney L. N.: Measurement of properties of brittle-matrix composites. British Ceramic Transactions, 92, 1-7 (1993). 
[8] De Baere I., Van Paepegema W., Degrieck J.: On the design of end tabs for quasi-static and fatigue testing of fibre-reinforced composites. Polymer Composites, 30, 381-390 (2008).

[9] Kawai M., Morishita M., Satoh H., Tomura S.: Effects of end-tab shape on strain field of unidirectional carbon/epoxy composite specimens subjected to off-axis tension. Composites Part A: Applied Science and Manufacturing, 28, 267-275 (1997).

DOI: $10.1016 / \mathrm{S} 1359-835 \mathrm{X}(96) 00122-4$

[10] Sun C. T., Chung I.: An oblique end-tab design for testing off-axis composite specimens. Composites, 24, 619-623 (1993).

DOI: 10.1016/0010-4361(93)90124-Q

[11] Curtis P.: CRAG test methods for the measurement of the engineering properties of fibre reinforced plastics. Technical Report 88012. Royal Aerospace Establishment, Farnborough (1988).

[12] De Baere I., Van Paepegem W., Quaresimin M., Degrieck J.: On the tension-tension fatigue behaviour of a carbon reinforced thermoplastic Part I: Limitations of the ASTM D3039/D3479 standard. Polymer Testing, 25, 625-632 (2011).

DOI: $10.1016 /$ j.polymertesting.2011.05.004

[13] De Baere I., Van Paepegem W., Hochard C., Degrieck J.: On the tension-tension fatigue behaviour of a carbon reinforced thermoplastic Part II: Evaluation of a dumbbell-shaped specimen. Polymer Testing, 30, 663-672 (2011).

DOI: 10.1016/j.polymertesting.2011.05.005

[14] Curtis D. C., Moore D. R., Slater B., Zahlan N.: Fatigue testing of multi-angle laminates of $\mathrm{CF} / \mathrm{PEEK}$. Composites, 19, 446-452 (1988). DOI: $10.1016 / 0010-4361(88) 90702-1$

[15] Xiao J., Bathias C.: Fatigue behaviour of unnotched and notched woven glass/epoxy laminates. Composites Science and Technology, 50, 141-148 (1994). DOI: 10.1016/0266-3538(94)90135-X

[16] Patrick M., Norman T. L.: Effect of notch on failure of two-dimensional $\pm 45^{\circ}$ triaxial braided textile composite materials. ASTM Journal of Composites Technology and Research, 16, 262-269 (1994).
[17] ASTM D7615/D7615M-11: Standard practice for openhole fatigue response of polymer matrix composite laminates (2011).

[18] Spearing S. M., Beaumont P. R. W.: Fatigue damage mechanics of composite materials. I: Experimental measurement of damage and post-fatigue properties. Composites Science and Technology, 44, 159-168 (1992). DOI: 10.1016/0266-3538(92)90109-G

[19] Liu C., Cheng L., Luan X., Li B., Zhou J.: Damage evolution and real-time non-destructive evaluation of 2D carbon-fiber/SiC-matrix composites under fatigue loading. Materials Letters, 62, 3922-3944 (2008). DOI: $10.1016 /$ j.matlet.2008.04.063

[20] Ogasawara T., Onta K., Ogihara S., Yokozeki T., Hara E.: Torsion fatigue behavior of unidirectional carbon/ epoxy and glass/epoxy composites. Composite Structures, 90, 482-489 (2009).

DOI: 10.1016/j.compstruct.2009.04.023

[21] Bailey P. B. S., Hoehl C., Jamshidi P., Cowan C., Squires S., Smith A. J.: Enhanced fatigue testing of composites. in 'Proceedings of the $19^{\text {th }}$ International Conference on Composite Materials, Montreal, Canada' 50335038 (2013).

[22] Toubal L., Karama M., Lorrain B.: Damage evolution and infrared thermography in woven composite laminates under fatigue loading. International Journal of Fatigue, 28, 1867-1872 (2006). DOI: $10.1016 /$ j.ijfatigue.2006.01.013

[23] Baker L. R., Webber J. M. B.: Thermoelastic stress analysis. Optica Acta: International Journal of Optics, 29, 555-563 (1982). DOI: $10.1080 / 713820858$

[24] Fruehmann R. K., Dulieu-Barton J. M., Quinn S.: Assessment of fatigue damage evolution in woven composite materials using infra-red techniques. Composites Science and Technology, 70, 937-946 (2010). DOI: $10.1016 /$ j.compscitech.2010.02.009 